ARTICLE

Received 2 May 2014 | Accepted 20 Aug 2014 | Published 6 Oct 2014

DOI: $10.1038 /$ ncomms 6048

OPEN

\title{
SKN-1 and Nrf2 couples proline catabolism with lipid metabolism during nutrient deprivation
}

Shanshan Pang ${ }^{1, \star}$, Dana A. Lynn ${ }^{1,2, \star}$, Jacqueline Y. Lo ${ }^{1,2}$, Jennifer Paek ${ }^{1} \&$ Sean P. Curran ${ }^{1,2,3}$

Mechanisms that coordinate different metabolic pathways, such as glucose and lipid, have been recognized. However, a potential interaction between amino acid and lipid metabolism remains largely elusive. Here we show that during starvation of Caenorhabditis elegans, proline catabolism is coupled with lipid metabolism by SKN-1. Mutation of alh-6, a conserved proline catabolic enzyme, accelerates fat mobilization, enhances the expression of genes involved in fatty acid oxidation and reduces survival in response to fasting. This metabolic coordination is mediated by the activation of the transcription factor SKN-1/Nrf2, possibly due to the accumulation of the alh- 6 substrate $\mathrm{P} 5 \mathrm{C}$, and also requires the transcriptional co-regulator MDT-15. Constitutive activation of SKN-1 induces a similar transcriptional response, which protects animals from fat accumulation when fed a high carbohydrate diet. In human cells, an orthologous alh-6 enzyme, ALDH4A1, is also linked to the activity of Nrf2, the human orthologue of SKN-1, and regulates the expression of lipid metabolic genes. Our findings identify a link between proline catabolism and lipid metabolism, and uncover a physiological role for SKN-1 in metabolism.

\footnotetext{
${ }^{1}$ Davis School of Gerontology, University of Southern California, 3715 McClintock Avenue, Los Angeles, California 90089, USA. ${ }^{2}$ Department of Molecular and Computational Biology, Dornsife College of Letters, Arts, and Sciences, University of Southern California, Los Angeles, California 90089, USA.

${ }^{3}$ Department of Biochemistry and Molecular Biology, Keck School of Medicine, University of Southern California, Los Angeles, California 90089 , USA. * These authors contributed equally to this work. Correspondence and requests for materials should be addressed to S.P.C. (email: spcurran@usc.edu).
} 
A nimals maintain energy homeostasis through the coordinated metabolism of available intracellular nutrients, including glucose, lipids and amino acids. To do so, animals employ complex but elegant molecular mechanisms to integrate the metabolism of these nutrients. In mice for example, under well-fed conditions, the liver $\mathrm{X}$ receptor integrates hepatic glucose metabolism and lipogenesis by acting as a glucose sensor $^{1}$. Glucose-mediated ChREBP activation in adipose tissue activates fatty acid synthesis, thus connecting glucose and lipid metabolism ${ }^{2}$. Although mechanisms such as these linking glucose and lipid metabolism have been well recognized, it remains largely elusive whether and how the metabolism of amino acids and lipids, two major nutrients for fasting responses, are coordinated.

During periods of nutrient deprivation, stored lipids and amino acids are used instead of dietary glucose to satisfy organismal energy requirements. Lipids are mobilized as an energy resource through lipolysis and fatty acid oxidation (FAO). Meanwhile, amino acids, another important energy resource, can either be directly oxidized or converted to glucose, and then oxidized by organs with an obligatory glucose requirement ${ }^{3}$. Based on the universal importance of these metabolic pathways during starvation, it is possible that their metabolism may be coupled together during fasting, and that this link would be well conserved.

SKN-1 is the worm homologue of the mammalian transcription factor Nrf2, both of which share a conserved cytoprotective function in the response to cellular electrophiles ${ }^{4}$. In addition, SKN-1 is a well-known longevity factor that is activated in many long-lived mutant backgrounds with altered metabolic homeostasis and is indispensable for the lifespan extension of those mutants ${ }^{5-8}$. Recently, we reported that gain-of-function mutations in $s k n-1$ lead to a starvation-like status in C. elegans and induce the expression of several metabolic genes ${ }^{9}$; however, the full extent to which SKN-1 participates in organismal physiology and metabolism remains unknown.

C. elegans is an established model for studying conserved pathways that govern lipid metabolism ${ }^{10-13}$. In this study, by using a $C$. elegans strain with a mutation in a conserved proline catabolic gene, we investigate the role of proline catabolism in the organismal response to fasting and discover that proline catabolism is coupled with fasting lipid utilization by the transcription factor SKN-1/Nrf2.

\section{Results}

Loss of alh-6 accelerates lipid mobilization during fasting. In a previous study, we identified mutations in the C. elegans gene alh6 , an evolutionarily conserved mitochondrial enzyme involved in the catabolism of proline (Supplementary Fig. 1a) ${ }^{14}$. In this study, we asked whether mutation of alh- 6 would affect lipid homeostasis. We first compared the fat content between wildtype and alh-6 mutant worms. When fed the standard Escherichia coli OP50 diet ad libitum, wild-type and alh-6 mutant worms store similar levels of intestinal fat as measured by Nile Red staining (Fig. 1a,b) or Oil Red O staining (Supplementary Fig. 1b). However, within a short $3 \mathrm{~h}$ exposure to starvation, alh-6 mutants rapidly mobilized intestinal lipids as compared with wild-type worms, which had yet to measurably use these nutrient stores (Fig. 1a,b and Supplementary Fig. 1b). Following a $18 \mathrm{~h}$ long-term starvation period, alh-6 mutants continued the hypermobilization of intestinal fat when compared with wild-type animals, which at this time point had also significantly depleted stored lipids (Fig. 1a,b). Thus, alh-6 mutants further enhance the mobilization of stored fat in response to food deprivation. This data indicates that alh-6 regulates lipid mobilization during starvation and implies that proline catabolism is coupled with lipid metabolism in response to nutrient depletion.
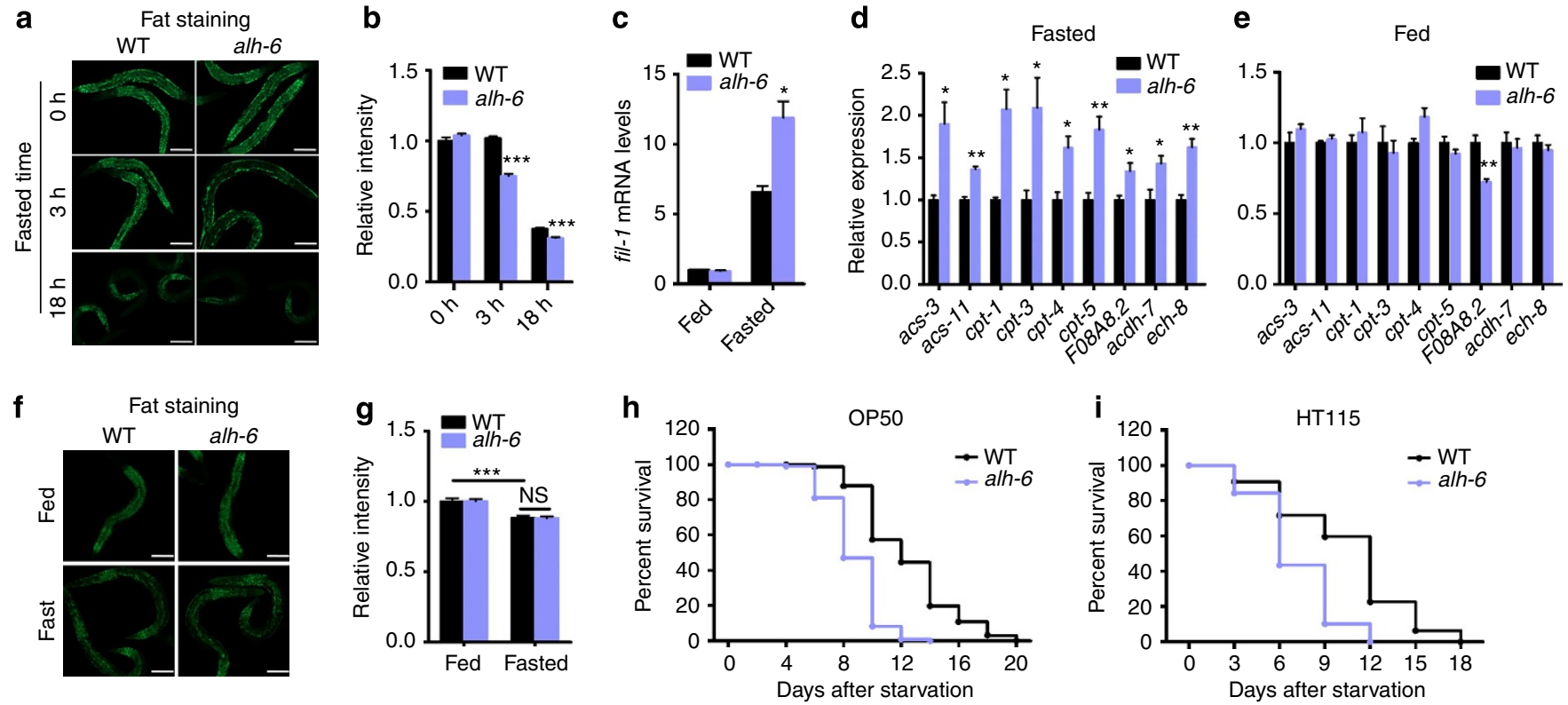

Figure 1 | Mutation of alh-6 enhances fat mobilization and the expression of FAO genes during starvation. (a,b) Nile Red staining of OP50 fed wild-type and alh-6 mutants in response to fasting. The representative images are shown in $\mathbf{a}$ (scale bar, $100 \mu \mathrm{m}$ ) and quantitative data are shown in $\mathbf{b}$ ( $n=12$ for $0 \mathrm{~h}$ of wild-type and alh-6 (lax105) mutants, $n=13$ for $3 \mathrm{~h}$ of alh-6 mutants, $n=9$ for other groups). (c) Expression of fil- 1 in response to $3 \mathrm{~h}$ fasting $(n=3)$. ( $(\mathbf{d}, \mathbf{e})$ Expression of FAO genes under $3 \mathrm{~h}$ fasted $(\mathbf{d})$ and well-fed $(\mathbf{e})$ conditions $(n=3)$. (f,g) Nile Red staining of wild type and alh-6 mutants fed HT115 in response to $3 \mathrm{~h}$ fasting. The representative images are shown in $\mathbf{f}$ (scale bar, $100 \mu \mathrm{m}$ ) and quantitative data are shown in $\mathbf{g}$ ( $n=8$ for fed wild type, $n=9$ for fed alh- 6 mutants and fasted wild type, $n=10$ for fasted alh- 6 mutants). (h,i) Survival rate of wild type and alh- 6 mutant worms during starvation when fed OP50 (h) or HT115 (i) diet before starvation. Data were presented as mean \pm s.e.m. $\left({ }^{\star} P<0.05,{ }^{\star \star} P<0.01,{ }^{\star \star \star} P<0.001\right.$, Student's $t$-test, versus wild-type controls under same treatment unless specifically indicated). 
We next examined the expression of genes involved in lipid metabolism. The expression levels of pod-2/ACC1 and fasn-1/ $F A S N$, the key enzymes in fatty acid synthesis, were comparably inhibited in wild-type and alh-6 mutant worms in response to fasting (Supplementary Fig. 1c). However, the expression of the fasting-induced lipase-1 (fil-1), a key lipolytic enzyme responsible for the C. elegans starvation response ${ }^{15}$, was significantly induced in alh-6 mutants during starvation above the measured increase in starved wild-type animals (Fig. 1c). Under fasted conditions, fat is used through mitochondrial and peroxisomal $\mathrm{FAO}^{16-19}$. We tested the expression of all annotated FAO enzymes in animals starved for $3 \mathrm{~h}$ (Supplementary Tables 1 and 2$)^{20}$. Consistent with the enhanced fat mobilization, alh-6 mutants exhibited increased expression of several FAO enzymes, specifically under fasted (Fig. 1d and Supplementary Table 1) but not well-fed conditions (Fig. 1e). These enzymes constitute several main steps of mitochondrial and peroxisomal FAO pathways ${ }^{20}$. Thus, fasted alh-6 mutants enhance lipid mobilization characterized by increased expression of genes involved in lipolysis and FAO but not de novo lipogenesis.

We identified several alh-6-sensitive FAO enzymes that were upregulated to enhance the wild-type fasting response (Supplementary Fig. 1d). In addition, we also discovered an increase in the expression of a subset of FAO genes that results in the derepression of targets that are inhibited in starved wild-type animals (Supplementary Fig. 1e) ${ }^{20}$. These data indicate that not all FAO enzymes are universally used under all starvation conditions, but rather some may specifically respond to the fasted state in the context of the alh-6 mutant background.

As mutations in alh-6 cause premature ageing in a dietdependent manner ${ }^{14}$, we asked whether the enhanced lipid mobilization phenotype identified above was also dependent on diet. Remarkably, the rapid depletion of intestinal lipid stores was abrogated when alh-6 mutants were raised on another common C. elegans diet, the E. coli K-12 strain HT115. Specifically, on this dietary regimen, alh-6 mutants exhibited comparable levels of fat mobilization in response to fasting (Fig. 1f,g) and showed no significant changes in the expression of FAO genes (Supplementary Fig. 1f) when compared with wild-type controls. Thus, the diet ingested before starvation establishes an organism's metabolic adaptation programme during food deprivation.

We also asked whether mutation of alh- 6 affected animal survival during fasting. We found that alh-6 mutants display significantly reduced animal survival in response to starvation (Fig. $1 \mathrm{~h}$ and Supplementary Table 3), further indicating that alh-6 is an important regulator of fasting adaptation. Intriguingly, the reduced survival of alh-6 mutant worms during fasting is not dependent on the diet before starvation (Fig. $1 \mathrm{i}$ and Supplementary Table 3), suggesting the role of alh-6 for survival during acute and long-term fasting are different.

SKN-1 mediates lipid metabolism responses in alh-6 mutants. The increased expression of FAO genes in fasted alh- 6 mutants indicates the existence of a transcriptional response that monitors and responds to perturbations in cellular proline metabolism. A role for the transcription factor SKN-1 has been documented under conditions of oxidative stress ${ }^{4}$ and lifespan extension ${ }^{5-8}$, where nutrient availability is either perceived as reduced or is actually reduced. Furthermore, we recently found that gain-offunction mutations in $s k n-1$ induce a starvation-like state ${ }^{9}$. As such, we proposed that a SKN-1-mediated transcriptional programme could mechanistically link proline and fatty acid metabolism. Under well-fed conditions, the expression of the SKN-1 transcriptional activity reporter gst-4p::GFP was similar between juvenile wild-type and alh-6 mutant worms (Fig. 2a). a
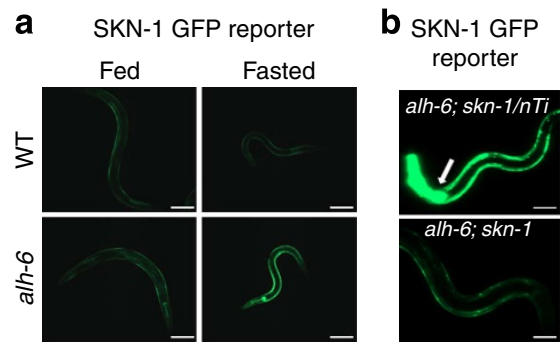

C

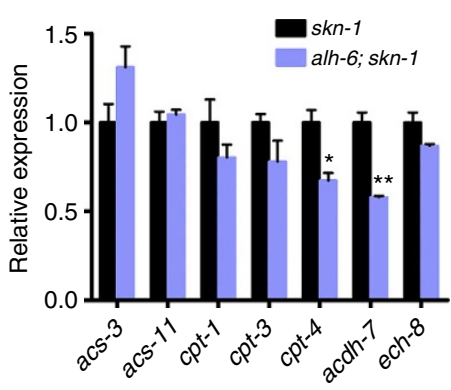

d Fasted

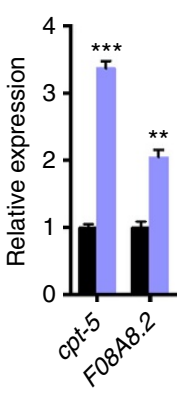

e

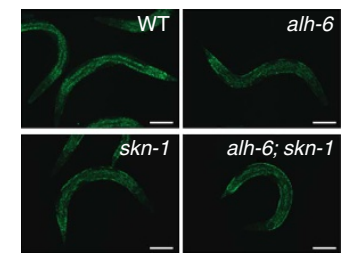

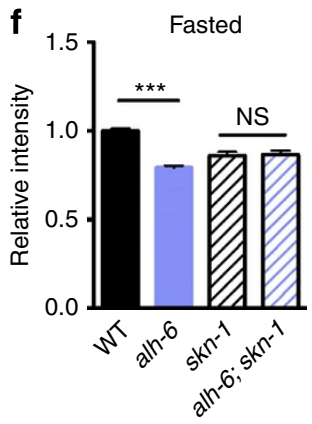

g 120

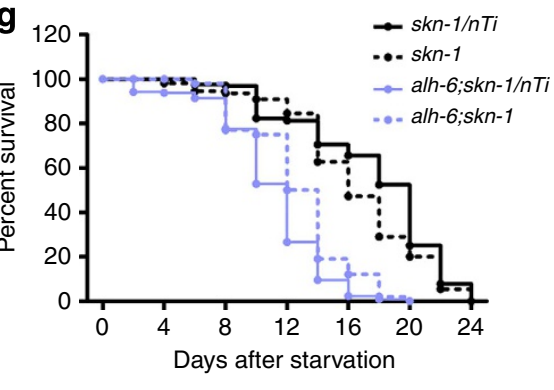

Figure 2 | SKN-1 coordinates proline and lipid metabolism during starvation of C. elegans. (a) Mutation of alh-6 activates gst-4p::GFP, a SKN-1 transcriptional activity reporter, during overnight fasting. Scale bar, $100 \mu \mathrm{m}$. (b) Mutation of $s k n-1$ abolished the activation of $g s t-4 p:: G F P$. The presence of the $s k n-1$ balancer is indicated by green fluorescent protein expression in the pharynx as pointed out by the arrow. Scale bar, $100 \mu \mathrm{m}$. (c,d) The increased expression of FAO genes in $3 \mathrm{~h}$ fasted alh-6 mutant is either dependent (c) or independent (d) on $s k n-1(n=3)$. (e,f) Nile Red staining of worms with indicated genotypes under $3 \mathrm{~h}$ fasted condition. The representative images are shown in $\mathbf{e}$ (scale bar, $100 \mu \mathrm{m}$ ) and quantitative data are shown in $\mathbf{f}$ ( $n=11$ for wild type, $n=10$ for other groups). (g) Effect of $s k n-1$ mutation on the starvation survival rate of alh-6 mutant worms. Data were presented as mean \pm s.e.m. $\left({ }^{\star} P<0.05,{ }^{\star \star} P<0.01,{ }^{\star \star \star} P<0.001\right.$, Student's $t$-test versus controls under same treatment unless specifically indicated). 
However, when starved, the SKN-1 reporter was dramatically activated in alh-6 mutants but not in wild-type controls (Fig. 2a). Furthermore, loss of SKN-1 function through null mutation substantially reduced the fasting-dependent activation of the SKN-1 reporter in alh-6 mutants (Fig. 2b). We conclude that alh6 mutants activate SKN-1 during food deprivation.

We next asked whether SKN-1 mediated the enhanced mobilization of stored lipids in fasted alh- 6 mutants. We found that seven out of nine FAO genes with increased expression in the fasted alh-6 mutants were no longer upregulated in the absence of SKN-1 (Fig. 2c). The expression of two FAO genes were still activated independently of SKN-1 in the alh-6 mutants during fasting (Fig. 2d), indicating the existence of other compensatory pathway(s) that function in parallel to SKN-1. Most importantly, a loss-of-function mutation in $s k n-1$ abrogated the enhanced depletion of intestinal lipid stores observed in alh-6 mutant worms after fasting (Fig. 2e,f), indicating an essential role for SKN-1 in mediating this fasting metabolic response. However, mutation of $s k n-1$ could not significantly reverse the reduced starvation survival rate of alh-6 mutant worms (Fig. $2 \mathrm{~g}$ ), further indicating the mechanistic differences between the lipid metabolism and survival responses in fasted alh- 6 mutants.

We previously reported that alh-6 mutations were capable of activating the SKN-1 reporter under fed conditions, but only after day 3 of the adult reproductive period ${ }^{14}$. Despite activation of SKN-1 at this time point in adult life, these alh-6 mutants did not induce a similar transcriptional change in FAO genes (Supplementary Fig. 2a) and do not reduce levels of stored fat (Supplementary Fig. 2b). These findings indicate a phenotypic difference between the same SKN-1-inducing mutation under different physiologic contexts, which suggests that the SKN-1mediated lipid response represents a specific metabolic response to the alh-6 mutation during starvation, and not merely an indirect side effect of global SKN-1 activation.
In our previous study, we also reported that accumulation of the alh-6 substrate $\mathrm{P} 5 \mathrm{C}$ and the subsequent generation of mitochondrial reactive oxygen species (ROS), such as hydrogen peroxide, are responsible for the premature ageing phenotype observed in adult alh-6 mutants ${ }^{14}$. Treatment with the antioxidant $\mathrm{N}$-acetylcysteine (NAC) completely abrogated the shortened lifespan of alh-6 mutants. As such, we evaluated a role for mitochondrial ROS in alh-6-mediated fasting lipid responses following NAC treatment. We found that although NAC treatment blocked the SKN-1 reporter activation induced by exposure to arsenite, an inducer of oxidative stress, NAC had no effect on the SKN-1 activation observed in fasted alh-6 mutants (Supplementary Fig. 2c). Moreover, NAC-treated alh-6 mutant worms still exhibited accelerated fat mobilization in response to fasting (Supplementary Fig. 2d). These data suggest that mitochondrial oxidative stress is not involved in SKN-1 activation and the enhanced lipid metabolism observed in fasted alh-6 worms. As SKN-1 can respond to multiple types of cellular stress, it is possible that additional, non-oxidative stress signals caused by $\mathrm{P} 5 \mathrm{C}$ accumulation are responsible for the observed SKN-1 activation and lipid changes.

SKN-1 protects against diet-induced fat accumulation. Subsequently, we tested whether $s k n$ - 1 gain-of-function mutations could induce a similar transcriptional response, and more importantly, if they result in a change in stored lipids. We discovered that well-fed $s k n-1$ gain-of-function mutant worms upregulated a large number of FAO genes (Fig. 3a), which is consistent with our previous observation that ad libitum-fed skn-1 gain-offunction animals behave as if they are starved ${ }^{9}$. Intriguingly, some FAO genes were found to be downregulated in $s k n-1$ gain-offunction mutants as compared with wild-type controls (Fig. 3b), further supporting the idea that unique FAO enzymes are differentially mobilized in response to particular metabolic
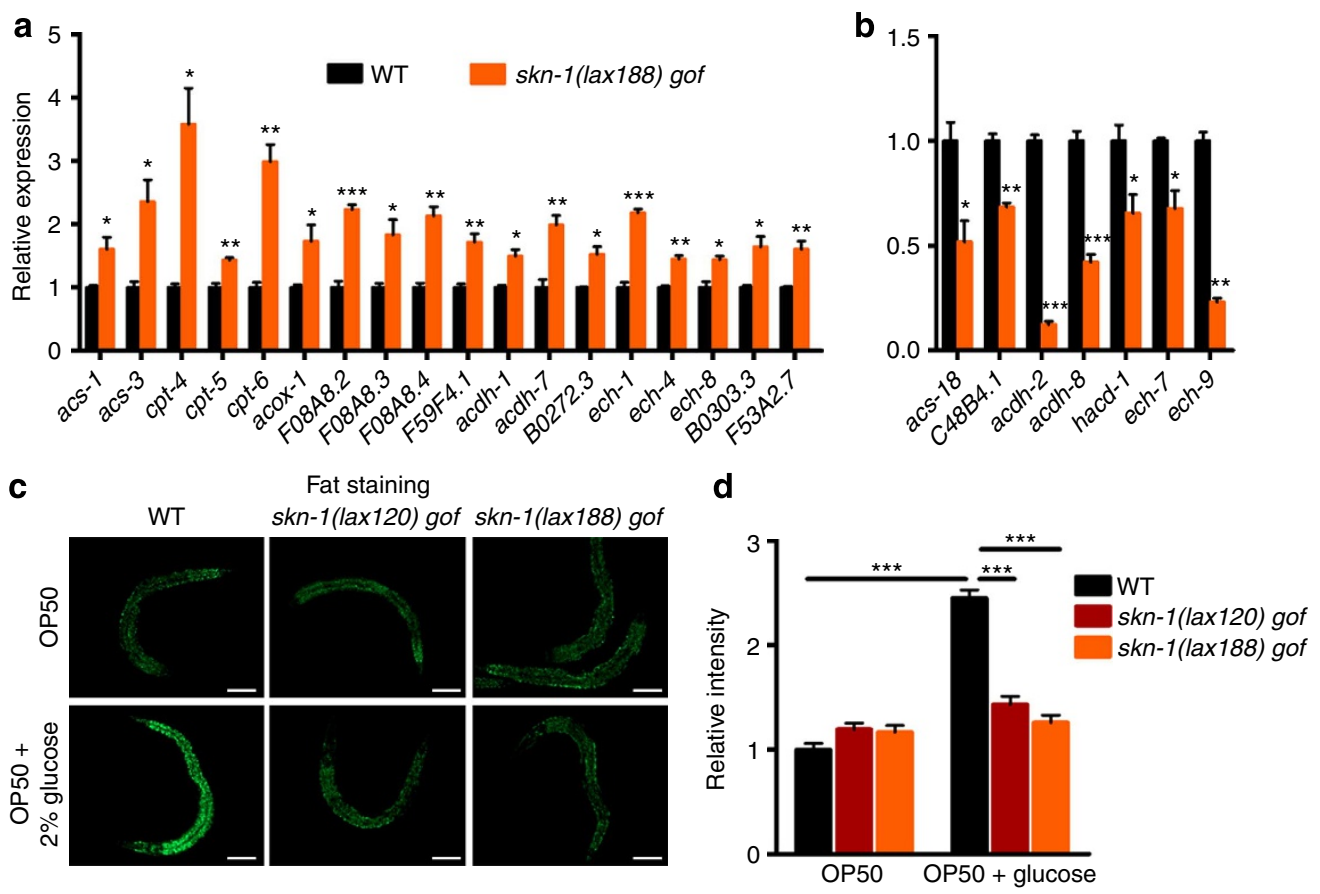

Figure 3 | Constitutive activation of SKN-1 protects animals from HCD-induced fat accumulation. (a,b) Expression of FAO genes that are upregulated (a) or downregulated (b) by skn-1(lax188) gain-of-function (gof) mutation $(n=3)$. (c,d) Nile Red staining of wild type, skn-1(lax120) and skn-1(lax188) gain-of-function mutants fed OP50 or OP50 plus $2 \%$ glucose. The representative images are shown in c (scale bar, $100 \mu \mathrm{m}$ ) and quantitative data are shown in $\mathbf{d}$ ( $n=5$ for wild type fed OP50, $n=10$ for wild type fed OP50 plus $2 \%$ glucose, $n=8$ for skn-1 (lax188) fed OP50, $n=7$ for other groups). Data were presented as mean \pm s.e.m. $\left({ }^{\star} P<0.05,{ }^{\star \star} P<0.01,{ }^{\star \star \star} P<0.001\right.$, Student's $t$-test versus controls under same treatment unless specifically indicated). 
stresses. Although, there was a larger set of lipid metabolism genes altered in the $s k n-1$ gain-of-function mutants, there was a clear overlap with the genes increased in the alh- 6 mutants during fasting (Supplementary Fig. 3a). This gene expression pattern indicates a SKN-1-dependent pathway for inducing an organismlevel metabolic response that is defined by the activation of fatty acid utilization pathways in both the $s k n-1$ gain-of-function mutants and SKN-1-activating alh-6 mutants under conditions of fasting. We then measured the fat content of those gain-of-function mutant worms. Although transcriptionally poised for increased oxidation of stored fat, well-fed skn-1 gain-of-function mutant animals exhibited relatively similar levels of fat content compared with well-fed wild-type controls as measured by Nile Red staining (Fig. 3c,d), and a minor decrease of fat as revealed by Oil Red O staining (Supplementary Fig. 3b). We hypothesized that the induction of FAO enzymatic activity in mutants with constitutive SKN-1 activation might only significantly impact lipid homeostasis, at the organismal level, under conditions of metabolic stress. We thus examined the function of constitutively activated SKN-1 in animals fed a high carbohydrate diet (HCD) ${ }^{21}$, which serves as model that mimics the diet-induced obesity observed in mammals. We found that addition of $2 \%$ glucose to the standard diet could significantly induce a $250 \%$ increase in stored intestinal fat in wild-type C. elegans, as compared with worms feeding on a normal diet (Fig. 3c,d and Supplementary Fig. 3b). Strikingly, when $s k n-1$ gain-of-function mutants were fed the HCD, they did not manifest this increased lipid phenotype (Fig. 3c,d and Supplementary Fig. 3b). These data suggest that constitutive SKN-1 activation can transcriptionally predispose animals to successfully cope with dietary insults, and that this adaptive capacity is capable of suppressing the lipid accumulation phenotype resulting from a HCD.

Aldh4a1 and Nrf2 regulate FAO genes in human cells. We next examined the possible conservation of the alh-6/skn-1 pathway in human cells. We first asked whether Nrf2, the human orthologue of SKN-1, also regulated the expression of FAO genes in human cells. Although Nrf2 activity has been linked to cancer cell metabolism and lipid biosynthesis in rodents ${ }^{22}$, its role in regulating FAO has not been established. We found that RNA interference (RNAi)-mediated knockdown of Nrf2 inhibited the expression of canonical Nrf2 target genes (Fig. 4a) and also several FAO genes in 293T cells (Fig. 4b), indicating that Nrf2 is a regulator of FAO genes in human cells. Next, we performed small interfering RNA (siRNA) knockdown of aldh4a1, the human orthologue of worm alh-6, and examined the effects on gene expression. Remarkably, aldh4a1 RNAi not only induced the expression of Nrf2 targets (Fig. 4c), which is indicative of Nrf2 activation, but also induced the expression of a subset of FAO genes (Fig. 4d). These data implicate that the SKN-1/ Nrf2-mediated regulatory axis between proline and lipid metabolism has functional conservation from invertebrates to humans.

MDT-15 co-regulates lipid metabolism with SKN-1. In light of the multitude of responses that are influenced by SKN-1/Nrf2, we predicted that the SKN-1/Nrf2 lipid metabolism response we identified would require additional transcriptional co-regulators. To identify possible co-regulators of SKN-1 in modulating lipid metabolism, we first screened an RNAi library targeting all annotated transcriptional regulators and DNA-binding proteins in C. elegans, looking for suppression of the SKN-1 reporter activation observed in the $s k n-1$ gain-of-function mutants 9 . We discovered that $m d t-15$ was required for SKN-1 reporter activation, as RNAi targeting $m d t-15$ significantly abolished the reporter activation (Fig. 5a). Moreover, in a complementary approach, we performed a classical ethyl methanesulfonate (EMS) mutagenesis screen for suppressors of the SKN-1 reporter activation in the $s k n$-1 gain-of-function mutant background. We isolated a single complementation group that mapped to the centre of LGIII and identified a Gly to Glu mutation in MDT-15 (Fig. 5b and Supplementary Fig. 4). MDT-15 is a transcriptional regulator of lipid metabolism ${ }^{23}$ and has been found to physically interact with SKN-1 (ref. 24). We then subsequently tested the role for MDT-15 in SKN-1-mediated lipid metabolism by examining the effect of $m d t-15$ RNAi on lipid gene expression in the skn-1 gain-of-function mutants. These mutants also display enhanced expression of FAO genes when raised on the control RNAi bacteria HT115 (Fig. 5c). However, it is notable that the gene expression changes observed are not identical to those when animals were fed the OP50 E. coli B diet (Fig. 3a,b), further supporting the diet-dependent response of SKN-1 function in lipid metabolism. RNAi knockdown of $m d t-15$ largely abolished the effects of $s k n-1$ gain-of-function mutation on FAO gene expression (Fig. 5c), suggesting MDT-15 is a critical cofactor for the transcription of these targets. Moreover, in the $m d t-15$ mutant background, alh- 6 mutant animals no longer exhibited the increased expression of FAO genes (Fig. 5d) or enhanced fat mobilization in response to fasting (Fig. 5e). Together, our results refine the molecular mechanisms by which SKN-1 and MDT-15
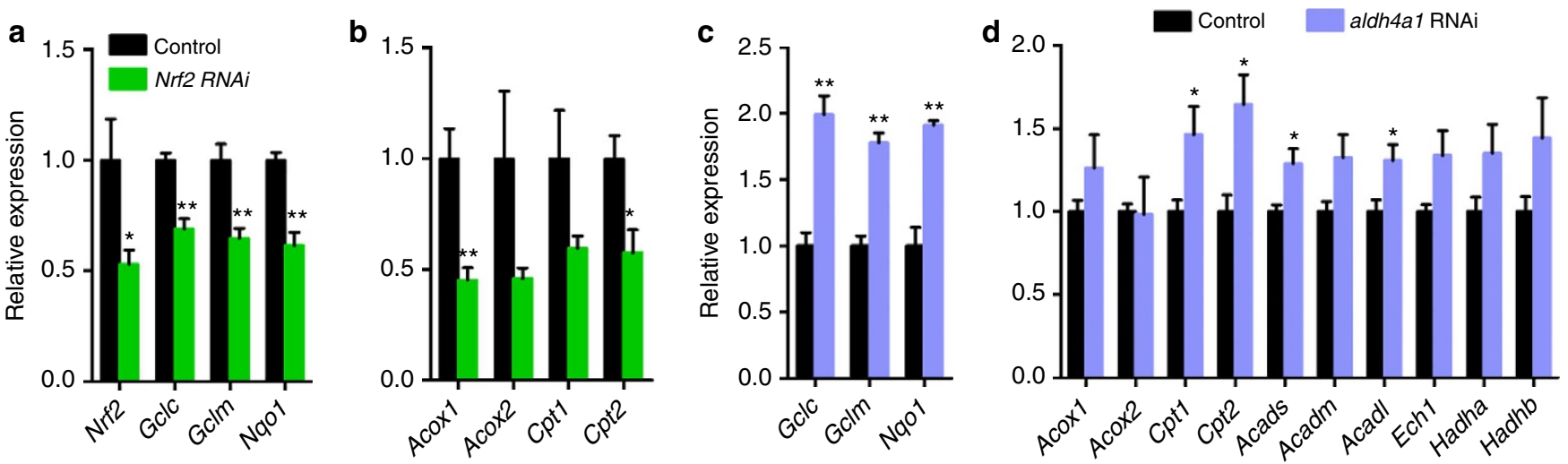

Figure 4 | Conserved regulation of Nrf2 activity and FAO genes by Aldh4a1. (a,b) Knockdown of Nrf2 inhibits expression of its canonical target genes (a) and FAO genes (b) ( $n=3$ for control, $n=5$ for Nrf2 RNAi). (c,d) Knockdown of aldh4a1 induces expression of Nrf2 target genes (c) ( $n=3)$ and FAO genes $(\mathbf{d})(n=6)$. Data were presented as mean \pm s.e.m. $\left({ }^{\star} P<0.05,{ }^{\star \star} P<0.01\right.$, Student's $t$-test versus controls). 
a
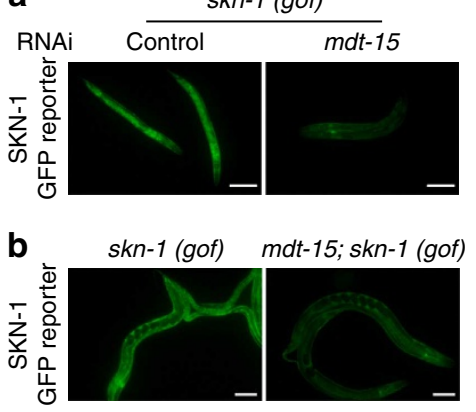

d

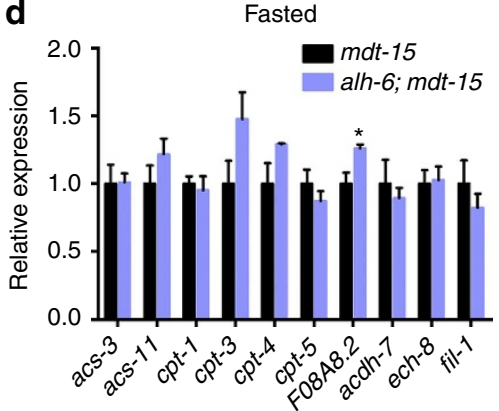

C

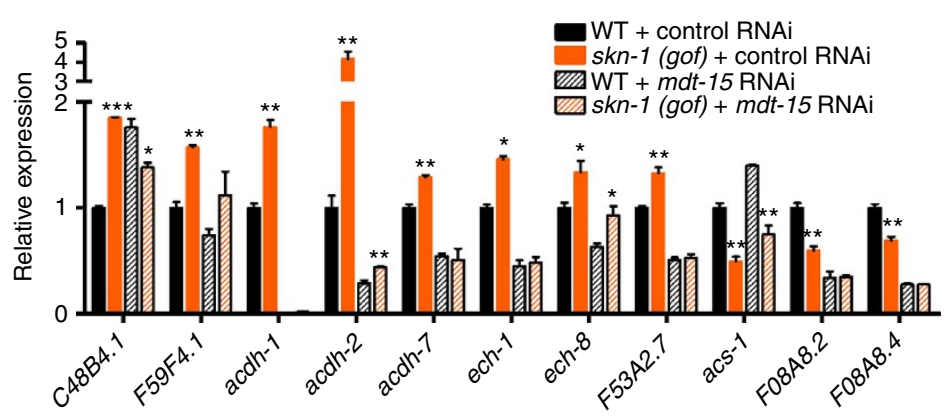

e

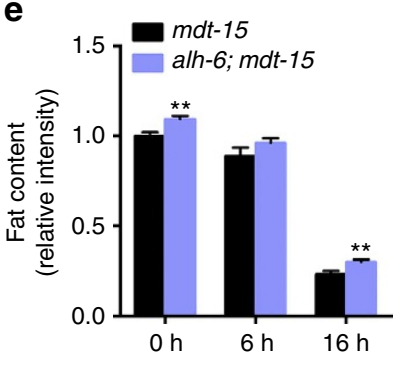

f Fasted

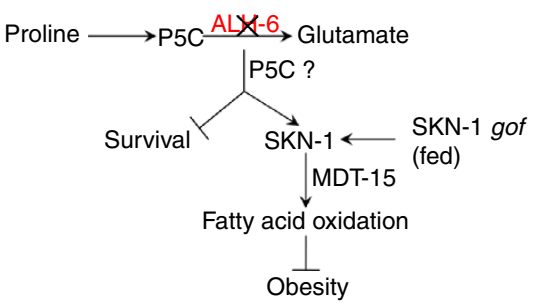

Figure 5 | MDT-15 is a co-factor for SKN-1-mediated lipid metabolism. (a,b) RNAi mediated knockdown (a) or point mutation (b) of mdt-15 abolishes the activation of gst-4p::GFP in skn-1 gain-of-function (gof) mutants skn-1 (lax188). Scale bar, $100 \mu \mathrm{m}$. (c) Expression of FAO genes that are regulated by skn-1 (gof)-fed HT115 bacteria containing L4440 control or mdt-15 RNAi plasmids ( $n=2$ for skn-1 (lax188)-fed control RNAi, $n=3$ for other groups). (d) The expression of alh-6-mediated FAO genes is largely dependent on $m d t-15(n=3)$. (e) Fat content of $m d t-15$ and alh-6; mdt- 15 mutants during starvation as measured by Nile Red staining ( $n=13$ for $0 \mathrm{~h}$ of $\mathrm{mdt}-15$ and $16 \mathrm{~h}$ of alh-6; mdt-15 mutants, $n=10$ for $6 \mathrm{~h}$ of $\mathrm{mdt}-15$ and alh-6; mdt-15 mutants, $n=11$ for $16 \mathrm{~h}$ of mdt-15 mutants, $n=12$ for $0 \mathrm{~h}$ of alh- 6 ; mdt-15 mutants). (f) Model: during fasting, mutation of the proline catabolic gene alh- 6 activates SKN-1, possibly through accumulation of metabolic intermediate P5C to mediate transcriptional programme for the induction of FAO genes, which also requires co-regulator MDT-15. Constitutively activated SKN-1 induces similar transcriptional changes in FAO genes that protect animals from diet-induced obesity. Data were presented as mean \pm s.e.m. $\left({ }^{\star} P<0.05,{ }^{\star \star} P<0.01,{ }^{\star \star \star} P<0.001\right.$, Student's $t$-test versus controls under same treatment unless specifically indicated).

cooperate to maintain lipid homeostasis and define MDT-15 as a co-regulator of SKN-1-dependent lipid metabolism.

\section{Discussion}

In this study, we reveal a novel link between proline and lipid metabolism, and identify a SKN-1/Nrf2-dependent mechanism that coordinates these two metabolic pathways (Fig. 5f). How does mutation of alh-6 lead to SKN-1 activation and lipid responses during fasting? A possible mechanism is that accumulation of the alh-6 substrate P5C induces SKN-1 activation and fat mobilization. A recent study in mammalian adipose cells has reported that during nutrient withdrawal, the activation of prodh, the enzyme producing $\mathrm{P} 5 \mathrm{C}$, can induce lipase expression $^{25}$. This finding supports the model for P5C as a conserved metabolic signal in activating SKN-1 and regulating fat mobilization during starvation. Generation and utilization of animals with mutations in or reduced expression of prodh, the P5C generating enzyme, will be valuable for testing this theory. Although Barbato et al. ${ }^{25}$ identified a role for ROS, this could represent the differences between our experimental models and readouts: apoptosis and inflammation in 3T3 cells versus organismal lipid depletion, or the inherent differences in responses for specific tissues. A more thorough understanding of the coordination of such responses will be of significant interest for future studies.

SKN-1 is an essential transcription factor mediating cellular stress responses, such as oxidative stress and immune defense.
Recent gene profile analyses indicate that SKN-1 may also be an important regulator of metabolism. In this study, we identify a physiological role for SKN-1 in metabolism, coordinating proline catabolism with lipid utilization during fasting. SKN-1 is thus a critical transcription factor that responds to diverse cellular stresses, including metabolic stress. Disruption of alh-6-dependent proline catabolism during fasting induces changes in the expression of several FAO genes, most of which are regulated in a SKN-1-dependent manner. Intriguingly, six of the seven SKN-1dependent genes we identified contain three to six conserved SKN-1-binding sites in their $2 \mathrm{~kb}$ promoters (Supplementary Fig. 3c); a SKN-1-binding site is generally found by chance only once in the same length of the genome $e^{4,26,27}$. This data indicates that some of these FAO genes may be direct targets of SKN-1.

We find that a subset of FAO genes, whose expression is inhibited by starvation in wild-type animals, is derepressed in fasted alh-6 mutant worms. This finding indicates physiological differences of the collection of FAO genes in the genome. We propose that $C$. elegans use unique FAO enzymes in response to distinct metabolic stress conditions: some metabolic enzymes can have overlapping functions and/or can be activated in response to specific cellular needs.

Another interesting finding of our study is that compromised alh-6-mediated proline catabolism regulates lipid metabolism during fasting in a diet-dependent manner. Although the response is triggered under a condition without food, our data suggests that dietary intake before food deprivation could predetermine an organism's response during starvation. The 
different nutritional composition between the OP50 and HT115 diets may lead to preferential use of specific energy substrates. We propose that, when fasted, animals previously fed an OP50 diet may rely more on mitochondrial alh-6 proline catabolism than those fed the HT115 diet. When alh- 6 is mutated, animals fed the OP50 diet might be more stressed when exogenous nutrients are no longer available. This condition thereby activates the lipid metabolism response through SKN-1 and MDT-15. Intriguingly, the diet consumed before fasting can have significant effects on mouse behaviour during food deprivation ${ }^{28}$, which suggests that dietary pre-determination of the adaptive response to starvation is also evolutionarily conserved.

Abnormal fat accumulation induced by diet underlies multiple metabolic diseases, such as obesity and type II diabetes. We and others show that a diet supplemented with high glucose can induce massive lipid accumulation in C. elegans, indicating the possibility of using this as a model to study diet-induced fat accumulation. In this study, we find that $s k n-1$ gain-of-function mutation protects animals against the increased lipid storage phenotype when fed a HCD. This finding implicates SKN-1 as a potential target for the treatment of abnormal lipid metabolism. Furthermore, Nrf2 can similarly regulate FAO genes in human cells revealing the evolutionary importance of this cellular metabolic response system. Thus, studies regarding the possible use of Nrf2 pathway agonists for regulating lipid metabolism and improving its related metabolic diseases will be of high clinical importance.

\section{Methods}

C. elegans growth conditions and strains. C. elegans were cultured using standard techniques at $20^{\circ} \mathrm{C}^{29}$. The following strains were used: wild-type N2 Bristol, SPC207: skn-1 (lax120), SPC227: skn-1 (lax188), SPC321: alh-6 (lax105), CL2166: gst4-p::gfp, SPC276: skn-1 (lax188); mdt-15 (lax225); gst4-p::gfp, VC1772: skn-1 (ok2315) IV/nTi[qIs51] (IV; V) and XA7702: $m d t-15$ (tm2182). Double or triple mutants were generated by standard genetic techniques.

Starvation assay. For starvation, synchronized L1 animals were added to nematode growth medium (NGM) plates seeded with indicated bacteria. After 2 days at $20^{\circ} \mathrm{C}, \mathrm{L} 4$ animals were collected, washed with M9 buffer at least three times and then subjected to fasting in M9 liquid with shaking for indicated time before collection for further analysis. Starvation survival assay was performed as previous described ${ }^{30}$. Briefly, gravid worms that did not experience starvation for at least two generations were used for egg preparation. After $24 \mathrm{~h}$, synchronized L1 animals were resuspended in M9 at a concentration of two worms per microlitre. Starvation culture was mixed by constant rocking. Every 2 days, a portion of animals was recovered on normal OP50-seeded NGM plates. Animals that resumed development were considered to be surviving.

Nile Red staining. Nile Red staining was performed as previously described ${ }^{31}$ Briefly, animals of indicated genotypes were collected, fixed in $40 \%$ isopropanol at room temperature for $3 \mathrm{~min}$ and stained in $3 \mu \mathrm{g} \mathrm{ml}^{-1}$ Nile Red working solution in dark for $2 \mathrm{~h}$. Worms were then washed with $\mathrm{M} 9$ for at least $30 \mathrm{~min}$, mounted on slides and imaged under the green fluorescent protein channel of microscope Zeiss Axio Imager with Zen software package. Fluorescent density was measured using ImageJ software. Approximately ten animals from each experiment $(n)$ were used to calculate the fluorescent density.

Oil Red $\mathbf{O}$ staining. Animals of indicated genotypes were collected and fixed in $1 \%$ formaldehyde in PBS for $10 \mathrm{~min}$. Next, samples were frozen and thawed three times with dry ice/ethanol bath. Worms were washed with PBS three times before staining with freshly prepared Oil Red O working solution. Worms were stained while rotating for $30 \mathrm{~min}$, washed again with PBS for $15 \mathrm{~min}$, mounted on slides and imaged under a bright-field illumination.

RNAi treatment. HT115 bacteria containing specific double stranded RNA-expression plasmids were seeded on NGM plates containing $5 \mathrm{mM}$ isopropyl$\beta$-D-thiogalactoside and $50 \mu \mathrm{g} \mathrm{ml}^{-1}$ carbenicillin. RNAi was induced at room temperature for $24 \mathrm{~h}^{32}$. Synchronized L1 animals were added to those plates to knockdown indicated genes.
Quantitative reverse transcription-PCR. Quantitative reverse transcription-PCR was performed as previously described ${ }^{14}$. Briefly, worms of the indicated genotype and stages were collected, washed in M9 buffer and then homogenized in Trizol reagent (Life Technologies). RNA was extracted according to the manufacturer's protocol. DNA contamination was digested with DNase I (New England Biolabs) and subsequently RNA was reverse-transcribed to complementary DNA by using the SuperScript III First-Strand Synthesis System (Life Technologies). Quantitative PCR was performed by using SYBR Green (BioRad). The expression levels of $s n b-1$ and actin were used to normalize samples in worms and human cells, respectively. Primer sequences listed in Supplementary Table 2.

Human cell culture. 293T cells were cultured in DMEM supplemented with $10 \%$ fetal bovine serum. At 50-70\% confluence, cells were transfected with control, Nrf2/Nfe2l2(s9492) or aldh4a1(s16484) Silencer Select (Life Technologies) siRNA by using Lipofectamine RNAiMax (Life Technologies). After $24 \mathrm{~h}$, cells were washed and collected in Trizol reagent (Invitrogen) for further RNA extraction. For examining the Nrf2 target genes in aldh4a1 siRNA experiment, cells were collected $48 \mathrm{~h}$ after transfection.

Statistical analysis. Data are presented as mean \pm s.e.m. Data were analysed by using unpaired Student's $t$-test. $P<0.05$ was considered as significant

\section{References}

1. Mitro, N. et al. The nuclear receptor LXR is a glucose sensor. Nature 445, 219-223 (2007).

2. Herman, M. A. et al. A novel ChREBP isoform in adipose tissue regulates systemic glucose metabolism. Nature 484, 333-338 (2012).

3. Cahill, Jr. G. F. Fuel metabolism in starvation. Annu. Rev. Nutr. 26, 1-22 (2006).

4. An, J. H. \& Blackwell, T. K. SKN-1 links C. elegans mesendodermal specification to a conserved oxidative stress response. Genes Dev. 17, 1882-1893 (2003).

5. Tullet, J. M. et al. Direct inhibition of the longevity-promoting factor SKN-1 by insulin-like signaling in C. elegans. Cell 132, 1025-1038 (2008).

6. Wang, J. et al. RNAi screening implicates a SKN-1-dependent transcriptional response in stress resistance and longevity deriving from translation inhibition. PLoS Genet. 6, e1001048 (2010).

7. Li, X. et al. Specific SKN-1/Nrf stress responses to perturbations in translation elongation and proteasome activity. PLoS Genet. 7, e1002119 (2011).

8. Bishop, N. A. \& Guarente, L. Two neurons mediate diet-restriction-induced longevity in C. elegans. Nature 447, 545-549 (2007).

9. Paek, J. et al. Mitochondrial SKN-1/Nrf mediates a conserved starvation response. Cell Metab. 16, 526-537 (2012).

10. Soukas, A. A., Kane, E. A., Carr, C. E., Melo, J. A. \& Ruvkun, G. Rictor/TORC2 regulates fat metabolism, feeding, growth, and life span in Caenorhabditis elegans. Genes Dev. 23, 496-511 (2009).

11. Ashrafi, K. et al. Genome-wide RNAi analysis of Caenorhabditis elegans fat regulatory genes. Nature 421, 268-272 (2003).

12. O'Rourke, E. J. \& Ruvkun, G. MXL-3 and HLH-30 transcriptionally link lipolysis and autophagy to nutrient availability. Nat. Cell Biol. 15, 668-676 (2013).

13. Watts, J. L. Fat synthesis and adiposity regulation in Caenorhabditis elegans. Trends Endocrinol. Metab. 20, 58-65 (2009).

14. Pang, S. \& Curran, S. P. Adaptive capacity to bacterial diet modulates aging in C. elegans. Cell Metab. 19, 221-231 (2014).

15. Jo, H., Shim, J., Lee, J. H., Lee, J. \& Kim, J. B. IRE-1 and HSP-4 contribute to energy homeostasis via fasting-induced lipases in C. elegans. Cell Metab. $\mathbf{9}$, 440-448 (2009).

16. Kersten, S. et al. Peroxisome proliferator-activated receptor alpha mediates the adaptive response to fasting. J. Clin. Invest. 103, 1489-1498 (1999).

17. Vega, R. B., Huss, J. M. \& Kelly, D. P. The coactivator PGC-1 cooperates with peroxisome proliferator-activated receptor alpha in transcriptional control of nuclear genes encoding mitochondrial fatty acid oxidation enzymes. Mol. Cell Biol. 20, 1868-1876 (2000).

18. Herzig, S. et al. CREB controls hepatic lipid metabolism through nuclear hormone receptor PPAR-gamma. Nature 426, 190-193 (2003).

19. Wolfrum, C., Asilmaz, E., Luca, E., Friedman, J. M. \& Stoffel, M. Foxa2 regulates lipid metabolism and ketogenesis in the liver during fasting and in diabetes. Nature 432, 1027-1032 (2004).

20. Van Gilst, M. R., Hadjivassiliou, H. \& Yamamoto, K. R. A Caenorhabditis elegans nutrient response system partially dependent on nuclear receptor NHR49. Proc. Natl Acad. Sci. USA 102, 13496-13501 (2005).

21. Schulz, T. J. et al. Glucose restriction extends Caenorhabditis elegans life span by inducing mitochondrial respiration and increasing oxidative stress. Cell Metab. 6, 280-293 (2007).

22. Sykiotis, G. P., Habeos, I. G., Samuelson, A. V. \& Bohmann, D. The role of the antioxidant and longevity-promoting Nrf2 pathway in metabolic regulation. Curr. Opin. Clin. Nutr. Metab. Care 14, 41-48 (2011). 
23. Taubert, S., Van Gilst, M. R., Hansen, M. \& Yamamoto, K. R. A Mediator subunit, MDT-15, integrates regulation of fatty acid metabolism by NHR-49-dependent and -independent pathways in C. elegans. Genes Dev. 20, 1137-1149 (2006).

24. Goh, G. Y. et al. The conserved Mediator subunit MDT-15 is required for oxidative stress responses in Caenorhabditis elegans. Aging Cell 13, 70-79 (2014).

25. Barbato, D. L. et al. Proline oxidase-adipose triglyceride lipase pathway restrains adipose cell death and tissue inflammation. Cell Death Differ. 21, 113-123 (2014).

26. Blackwell, T. K., Bowerman, B., Priess, J. R. \& Weintraub, H. Formation of a monomeric DNA binding domain by Skn-1 bZIP and homeodomain elements. Science 266, 621-628 (1994).

27. Oliveira, R. P. et al. Condition-adapted stress and longevity gene regulation by Caenorhabditis elegans SKN-1/Nrf. Aging Cell 8, 524-541 (2009).

28. Lavin, D. N. et al. Fasting induces an anti-inflammatory effect on the neuroimmune system which a high-fat diet prevents. Obesity. (Silver Spring) 19, 1586-1594 (2011)

29. Brenner, S. The genetics of Caenorhabditis elegans. Genetics 77, 71-94 (1974).

30. Artyukhin, A. B., Schroeder, F. C. \& Avery, L. Density dependence in Caenorhabditis larval starvation. Sci. Rep. 3, 2777 (2013).

31. Pino, E. C., Webster, C. M., Carr, C. E. \& Soukas, A. A. Biochemical and high throughput microscopic assessment of fat mass in Caenorhabditis elegans. J. Vis. Exp. 73 doi:10.3791/50180 (2013).

32. Kamath, R. S. et al. Systemic functional analysis of the Caenorhabditis elegans genome using RNAi. Nature 421, 231-237 (2003).

\section{Acknowledgements}

We thank E. Roh and L. Thomas for technical assistance, and members of the Curran laboratory for discussions. The $293 \mathrm{~T}$ cell line was kindly provided by Dr Deborah
Johnson. Some strains were provided by the CGC, which is funded by the NIH Office of Research Infrastructure Programs (P40 OD010440). S.P.C. is an Ellison Medical Foundation New Scholar in Aging; this work was funded by the NIH (AG032308), the Ellison Medical Foundation and the American Federation of Aging Research.

\section{Author contributions}

S.P. and S.P.C. designed the study; S.P., D.A.L., J.Y.L., J.P. and S.P.C. performed the experiments; S.P., D.A.L. and S.P.C. analysed data and wrote the manuscript.

\section{Additional information}

Supplementary Information accompanies this paper at http://www.nature.com/ naturecommunications

Competing financial interests: The authors declare no competing financial interests.

Reprints and permission information is available online at http://npg.nature.com/ reprintsandpermissions/

How to cite this article: Pang, S. et al. SKN-1 and Nrf2 couples proline catabolism with lipid metabolism during nutrient deprivation. Nat. Commun. 5:5048 doi: $10.1038 /$ ncomms6048 (2014).

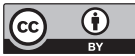

This work is licensed under a Creative Commons Attribution 4.0 International License. The images or other third party material in this article are included in the article's Creative Commons license, unless indicated otherwise in the credit line; if the material is not included under the Creative Commons license, users will need to obtain permission from the license holder to reproduce the material. To view a copy of this license, visit http://creativecommons.org/licenses/by/4.0/ 